\title{
Investigation of methylenetetrahydrofolate reductase tagging polymorphisms with colorectal cancer in Chinese Han population
}

\author{
Sheng Zhang ${ }^{1, *}$, Shuchen Chen ${ }^{2, *}$, Yu Chen ${ }^{3,4, *}$, Mingqiang Kang ${ }^{2}$, Chao Liu ${ }^{5}$, Hao \\ Qiu', Yafeng Wang7 and Weifeng Tang ${ }^{2,5}$ \\ ${ }^{1}$ Department of General Surgery, Changzhou No. 3 People's Hospital, Changzhou, Jiangsu Province, China \\ ${ }^{2}$ Department of Thoracic Surgery, Affiliated Union Hospital of Fujian Medical University, Fuzhou, Fujian Province, China \\ ${ }^{3}$ Department of Medical Oncology, Fujian Provincial Cancer Hospital, Fujian Medical University Cancer Hospital, Fuzhou, \\ Fujian Province, China \\ ${ }^{4}$ Fujian Provincial Key Laboratory of Translational Cancer Medicine, Fuzhou, Fujian Province, China \\ ${ }^{5}$ Department of Cardiothoracic Surgery, Affiliated People's Hospital of Jiangsu University, Zhenjiang, Jiangsu Province, China \\ ${ }^{6}$ Department of Immunology, School of Medicine, Jiangsu University, Zhenjiang, Jiangsu Province, China \\ ${ }^{7}$ Department of Cardiology, The People's Hospital of Xishuangbanna Dai Autonomous Prefecture, Jinghong, Yunnan Province, \\ China \\ "These authors have contributed equally to this work \\ Correspondence to: Weifeng Tang, email: twf001001@126.com \\ Yafeng Wang, email: wangyafeng0910@126.com \\ Keywords: polymorphism, MTHFR, colorectal cancer, tagging, susceptibility \\ Received: January 27, $2017 \quad$ Accepted: June 04, $2017 \quad$ Published: June 29, 2017 \\ Copyright: Zhang et al. This is an open-access article distributed under the terms of the Creative Commons Attribution License 3.0 \\ (CC BY 3.0), which permits unrestricted use, distribution, and reproduction in any medium, provided the original author and source \\ are credited.
}

\section{ABSTRACT}

The aim of this case-control study was to assess the relationship between the tagging polymorphisms in methylenetetrahydrofolate reductase (MTHFR) gene and the susceptibility to colorectal cancer (CRC) in a Chinese Han population. A customby-design 48-Plex SNPscan Kit was used to determine the genotypes of MTHFR rs3753584 T>C, rs9651118 T>C, rs1801133 G>A, rs4846048 A>G and rs4845882 G>A polymorphisms in 387 CRC patients and 1,536 non-cancer controls. The results revealed that MTHFR rs1801133 G>A polymorphism was associated with a decreased risk of overall CRC. While MTHFR rs4845882 G>A polymorphism conferred an increased risk to overall CRC. In a stratified analysis by CRC region, we found MTHFR rs3753584 T>C and rs9651118 T>C polymorphisms were associated with the increased risk of colon cancer. In addition, a significantly increased risk of rectum cancer associated with MTHFR rs3753584 T>C polymorphism was overt. However, MTHFR rs1801133 G>A polymorphism conferred a decreased risk to colon cancer. In conclusion, findings of the present study reveal that the tagging polymorphisms in MTHFR gene ( $r s 3753584$ T $>C$, rs9651118 $T>C$ and rs4845882 G $>A$ ) are associated with the increased risk of CRC. However, MTHFR rs1801133 G>A polymorphism confers a decreased risk to CRC. Additional studies with larger sample size are needed to confirm these findings.

\section{INTRODUCTION}

Colorectal cancer (CRC) is the fourth most frequent type of malignancy among females and the fifth most frequent type among males in China, accounting for
160,600 and 215,700 cases in 2015 , respectively [1]. The CRC morbidity is shooting up in developing countries including China $[1,2]$; nevertheless, CRC etiology remains unknown. Risk factors, such as advanced age, family history of $\mathrm{CRC}$, benign adenomatous polyp, 
inflammatory bowel disease, drinking, smoking, being physically inactive, low intake of fruits and vegetables and high intake of dietary fat, may play important roles in the occurrence and the development of CRC [3-9]. Accumulating evidences indicated that besides environmental factors and individual lifestyle, some genetic factors might be related to CRC etiology.

Several studies have found association between circulating or dietary folate level and the risk of CRC [10-12]. A long induction period deserves close attention to the study of CRC because the course from normal cells of the rectum and colon, to microadenomas, to macroadenomas, and eventually to adenocarcinomas, experiences over a long duration, approximately 30-40 years [13]. The presence of the long induction period between reduced risk of CRC and adequate folate status is evident in both epidemiologic and molecular mechanistic studies [14]. The lower folate levels lead to an increasing rate of DNA hypomethylation and uracil misincorporation even in non-neoplastic, normal-appearing tissue of cases [14]. Folate is reduced to tetrahydrofolate (THF) to participate in one-carbon metabolism [15]. Folate metabolism is accommodated by several enzymes. Methylenetetrahydrofolate reductase (MTHFR) is the key enzyme [16-18]. MTHFR is involved in DNA methylation, repair and synthesis [19]. DNA strand break and repair, and impaired DNA methylation have been associated with folate deficiency and CRC.

MTHFR gene locates on 1p36.22. The gene encodes a $74.6-\mathrm{kDa}$ protein containing 656 amino acids. MTHFR is also linked to purine synthesis; therefore, plays a vital role in DNA synthesis by the provision of essential nucleotides [18]. Single nucleotide polymorphisms (SNPs) of MTHFR are considered as a potential biomarker which may influence $\mathrm{CRC}$ risk. Therefore, we carried out this case-control study in a Chinese Han population to determine whether MTHFR tagging SNPs (rs3753584 T $>$ C, rs9651118 T $>$ C, rs1801133 $\mathrm{G}>\mathrm{A}$, rs4846048 $\mathrm{A}>\mathrm{G}$ and $\mathrm{rs} 4845882 \mathrm{G}>\mathrm{A}$ ) were associated with the risk of CRC.

\section{RESULTS}

\section{Demographic characteristics}

The frequency distributions of age, sex, smoking status and drinking habit information for the 387 patients with CRC (mean \pm SD age, $60.21 \pm 12.48$ years) and 1,536 controls (mean \pm SD age, $60.82 \pm 8.85$ years) are shown in Table 1. The age and sex of the CRC patients and controls were well-matched. The differences of smoking status and drinking habit between CRC and non-cancer controls were not statistically significant $(P \geq 0.05)$ (Table 1). Tumor grade was defined as high (well differentiated), medium (moderately differentiated); and low (poorly differentiated). Two experienced doctors independently assessed disease stage according to the AJCC criteria (2010). Finally, 196 cases with stage I/II and 191 with stage III/IV were included. Among the CRC patients, 218 were rectum cancer and 169 were colon cancer. And the primary information for MTHFR polymorphisms is summarized in Table 2.

\section{Association of MTHFR $\operatorname{rs} 3753584 \mathrm{~T}>\mathrm{C}$, rs9651118 $T>C, \operatorname{rs} 1801133 \mathrm{G}>A$, rs4846048 $A>G$ and $\mathrm{rs} 4845882 \mathrm{G}>\mathrm{A}$ polymorphisms with $\mathrm{CRC}$ patients}

The genotypes of MTHFR rs3753584 T $>\mathrm{C}$, rs9651118 $\mathrm{T}>\mathrm{C}$, rs1801133 $\mathrm{G}>\mathrm{A}$, rs4846048 $\mathrm{A}>\mathrm{G}$ and $\mathrm{rs} 4845882 \mathrm{G}>\mathrm{A}$ polymorphisms in CRC patients and non-cancer controls are summarized in Table 3. The observed genotype frequencies for the five MTHFR tagging SNPs in control group were all in $\operatorname{HWE}(P=0.134,0.082,0.733,0.164$ and 0.747 for MTHFR rs9651118 T $>$ C, rs4846048 A $>$ G, rs4845882 G $>$ A, rs3753584 $\mathrm{T}>\mathrm{C}$ and $\mathrm{rs} 1801133 \mathrm{G}>\mathrm{A}$, respectively), which suggested good homogeneity within the present study participants. Four CRC patients (1.03\%) and three controls $(0.19 \%)$ could not be genotyped for poor DNA quantity and/or quality. Overall, we found no statistically significant difference in genotype distribution of MTHFR rs3753584 $\mathrm{T}>\mathrm{C}$, rs9651118 $\mathrm{T}>\mathrm{C}$ and $\mathrm{rs} 4846048 \mathrm{~A}>\mathrm{G}$ polymorphisms among CRC patients and controls (Table 4).

When compared with MTHFR rs1801133 GG genotype, MTHFR rs1801133 AA genotype was associated with a decreased risk of CRC (crude OR $=0.56,95 \% \mathrm{CI}$ : $0.37-0.84, P=0.006)$. When compared with MTHFR rs1801133 GG/GA genotype, MTHFR rs1801133 AA genotype was also associated with a decreased risk of CRC (crude OR $=0.60,95 \%$ CI: $0.40-0.89, P=0.011$ ). Adjustment for multiple factors (age, sex, smoking and drinking), the results were not essentially changed (AA vs. GG: adjusted $\mathrm{OR}=0.56,95 \% \mathrm{CI}: 0.37-0.84, P=$ 0.005 ; AA vs. GG/GA: OR $=0.60,95 \%$ CI: $0.40-0.89, P$ $=0.011$; Table 4).

When compared with MTHFR rs4845882 GG genotype, a MTHFR rs4845882 AA genotype increased the risk of $\mathrm{CRC}$ (crude OR $=1.65,95 \% \mathrm{CI}$ : $1.03-2.66$, $P=0.039$ ). When compared with MTHFR rs4845882 GG/GA genotype, MTHFR rs4845882 AA genotype was associated with an increased risk of CRC (crude OR $=1.65,95 \% \mathrm{CI}: 1.03-2.63, P=0.038)$. Adjustment for multiple factors (age, sex, smoking and drinking), the results were not essentially changed (AA vs. GG: adjusted OR $=1.6495 \%$ CI: $1.02-2.65, P=0.043$; AA vs. GG/GA: $\mathrm{OR}=1.63,95 \% \mathrm{CI}: 1.01-2.60, P=0.044$; Table 4 ).

\section{Association of MTHFR rs3753584 T $>C$, rs9651118 $\mathrm{T}>\mathrm{C}, \mathrm{rs} 1801133 \mathrm{G}>\mathrm{A}, \mathrm{rs} 4846048 \mathrm{~A}>\mathrm{G}$ and $r \mathbf{4 8 4 5 8 8 2} \mathrm{G}>\mathrm{A}$ polymorphisms with $\mathrm{CRC}$ in a stratification group}

To determine whether the effect of MTHFR polymorphisms was influenced by $\mathrm{CRC}$ region, we 
Table 1: Distribution of selected demographic variables and risk factors in colorectal cancer cases and controls

\begin{tabular}{|c|c|c|c|c|c|}
\hline \multirow{2}{*}{ Variable } & \multicolumn{2}{|c|}{ Cases $(n=387)$} & \multicolumn{2}{|c|}{ Controls $(n=1,536)$} & \multirow{2}{*}{$P^{\mathrm{a}}$} \\
\hline & $\mathbf{n}$ & $\%$ & $\mathbf{n}$ & $\%$ & \\
\hline Age (years) & $60.21( \pm 12.48)$ & & $60.82( \pm 8.85)$ & & 0.272 \\
\hline Age (years) & & & & & 0.502 \\
\hline$<61$ & 186 & 48.06 & 709 & 46.16 & \\
\hline$\geq 61$ & 201 & 51.94 & 827 & 53.84 & \\
\hline Sex & & & & & 0.213 \\
\hline Male & 236 & 60.98 & 989 & 64.39 & \\
\hline Female & 151 & 39.02 & 547 & 35.61 & \\
\hline Smoking status & & & & & 0.505 \\
\hline Never & 270 & 69.77 & 1098 & 71.48 & \\
\hline Ever & 117 & 30.23 & 438 & 28.52 & \\
\hline Alcohol use & & & & & 0.058 \\
\hline Never & 335 & 78.55 & 1381 & 89.91 & \\
\hline Ever & 52 & 21.45 & 155 & 10.09 & \\
\hline \multicolumn{6}{|l|}{ Site of tumor } \\
\hline Colon cancer & 169 & 43.67 & & & \\
\hline Rectum cancer & 218 & 56.33 & & & \\
\hline \multicolumn{6}{|l|}{$\begin{array}{l}\text { Degree of } \\
\text { differentiation }^{\mathrm{b}}\end{array}$} \\
\hline Low & 56 & 16.28 & & & \\
\hline Medium & 261 & 75.87 & & & \\
\hline High & 27 & 7.85 & & & \\
\hline \multicolumn{6}{|l|}{$\begin{array}{l}\text { Lymph node } \\
\text { status }\end{array}$} \\
\hline Positive & 177 & 45.74 & & & \\
\hline Negative & 210 & 54.26 & & & \\
\hline \multicolumn{6}{|l|}{ TMN stage } \\
\hline $\mathrm{I}+\mathrm{II}$ & 196 & 50.65 & & & \\
\hline $\mathrm{III}+\mathrm{IV}$ & 191 & 49.35 & & & \\
\hline
\end{tabular}

awo-sided $\chi^{2}$ test and Student $t$ test.

performed a stratified analysis. For MTHFR rs3753584 $\mathrm{T}>\mathrm{C}$ polymorphism, we found this polymorphism was associated with an increased risk of rectum cancer (CC+TC vs. TT: adjusted OR $=1.44,95 \% \mathrm{CI}=1.04-2.00$, $P=0.026$; TC vs. TT: adjusted OR $=1.44,95 \% \mathrm{CI}=1.04$ 2.00, $P=0.030)$ and of colon cancer (CC vs. TT+TC: adjusted $\mathrm{OR}=3.74,95 \% \mathrm{CI}=1.31-10.64, P=0.014$; CC vs. TT: adjusted $\mathrm{OR}=3.63,95 \% \mathrm{CI}=1.27-10.38$, $P=0.016)$. For MTHFR rs9651118 T $>$ C polymorphism, we found MTHFR rs9651118 CC genotypes might be associated with an increased risk of colon cancer (CC vs. TT+TC: adjusted $\mathrm{OR}=1.53,95 \% \mathrm{CI}=1.01-2.31, P$ $=0.044$ ). However, we found MTHFR rs1801133 G>A polymorphism was associated with a decreased risk of colon cancer (AA vs. GG+GA: adjusted OR $=0.52,95 \%$ $\mathrm{CI}=0.28-0.95, P=0.035$; AA vs. GG: adjusted $\mathrm{OR}=$ $0.46,95 \% \mathrm{CI}=0.25-0.86, P=0.015)$. Other comparisons are presented in Table 4. 
Table 2: Primary information for $M T H F R$ polymorphisms (rs3753584 T $>$ C, rs9651118 T $>$ C, rs1801133 G $>$ A, rs4846048 $\mathrm{A}>\mathrm{G}$ and $\mathbf{r s 4 8 4 5 8 8 2} \mathrm{G}>\mathrm{A})$

\begin{tabular}{|c|c|c|c|c|c|}
\hline Genotyped SNPs & rs3753584 T $>C$ & rs9651118 $\mathrm{T}>\mathrm{C}$ & $\operatorname{rs1801133~G>A}$ & rs4846048 A>G & rs4845882 G>A \\
\hline Chromosome & 1 & 1 & 1 & 1 & 1 \\
\hline Function & NearGene-5 & Intron & Missense & Intron & Intron \\
\hline $\begin{array}{l}\text { Chr Pos (Genome } \\
\text { Build 36.3) }\end{array}$ & 11787173 & 11784801 & 11778965 & 11768839 & 11765754 \\
\hline $\begin{array}{l}\text { MAF for Chinese } \\
\text { in database }\end{array}$ & 0.093 & 0.382 & 0.439 & 0.105 & 0.198 \\
\hline $\begin{array}{l}\text { MAF in our } \\
\text { controls }(\mathrm{n}= \\
1,536)\end{array}$ & 0.109 & 0.379 & 0.356 & 0.096 & 0.209 \\
\hline $\begin{array}{l}P \text { value for } \\
\mathrm{HWE}^{\mathrm{b}} \text { test in our } \\
\text { controls }\end{array}$ & 0.164 & 0.134 & 0.747 & 0.082 & 0.733 \\
\hline $\begin{array}{l}\text { Genotyping } \\
\text { method }\end{array}$ & SNPscan & SNPscan & SNPscan & SNPscan & SNPscan \\
\hline $\begin{array}{l}\% \text { Genotyping } \\
\text { value }\end{array}$ & $99.64 \%$ & $99.64 \%$ & $99.64 \%$ & $99.64 \%$ & $99.64 \%$ \\
\hline
\end{tabular}

${ }^{a} \mathrm{MAF}$ : minor allele frequency;

${ }^{b}$ HWE: Hardy-Weinberg equilibrium.

\section{The power of the present study $(\alpha=0.05)$}

For MTHFR rs3753584 T $>\mathrm{C}$, the power value was 0.634 in homozygote model and 0.651 in recessive model among colon cancer group, and 0.586 in homozygote model and 0.614 in recessive model among rectum cancer group. In addition, for MTHFR rs $9651118 \mathrm{~T}>\mathrm{C}$, the power value was 0.527 in recessive model among colon cancer group. Moreover, for MTHFR rs $1801133 \mathrm{G}>\mathrm{A}$, the power value was 0.815 in homozygote model and 0.748 in recessive model among overall CRC group, and 0.705 in homozygote model and 0.565 in recessive model among colon cancer group. For MTHFR rs4845882 G>A, the power value was 0.537 in homozygote model and 0.553 in recessive model among overall CRC group, and 0.494 in homozygote model and 0.540 in recessive model among rectum cancer group as well.

\section{DISCUSSION}

Recently, CRC incidence and related mortality are increasing rapidly worldwide. The individual's susceptibility to CRC may be influenced by some environmental exposure and genetic factors. Recently, many case-control studies have been directed towards the association between MTHFR polymorphisms and CRC risk. However, the sample size of most studies was relatively small. Here, we attempt to assess the association between MTHFR tagging SNPs (rs3753584 T>C, rs9651118 T $>$ C, rs1801133 G $>$ A, rs4846048 $\mathrm{A}>\mathrm{G}$ and rs4845882 $\mathrm{G}>\mathrm{A}$ ) and susceptibility of CRC. Our results indicated several MTHFR tagging polymorphisms could affect the risk of CRC.

MTHFR rs1801133 G>A polymorphism leads to an amino acid transformation (alanine $\rightarrow$ valine at 226 position of MTHFR protein). The role of MTHFR enzyme is remethylation of homocysteine to methionine. Polymorphisms in MTHFR gene are corrected with the deficiency of MTHFR enzyme activity. Compared with rs1801133 GG homozygote, MTHFR rs1801133 AA homozygote decreases $70 \%$ of the enzyme activity and MTHFR rs1801133 GA heterozygote loss 35\% of enzymatic function. This transformation may increase the plasma homocysteine (Hcy) concentration and decreases the plasma folic acid concentration [26]. Recently, several meta-analyses indicated that MTHFR rs $1801133 \mathrm{G}>\mathrm{A}$ polymorphism decreased the risk of CRC in Asians [27, 28]. MTHFR rs 1801133 $\mathrm{G}>\mathrm{A}$ polymorphism locates on the NH2-terminal catalytic domain. In addition, MTHFR rs1801133 $\mathrm{G}>\mathrm{A}$ polymorphism increases the availability of 5 , 10-methylenetetrahydrofolate for DNA synthesis [29, 30], which may partially explain the protective factor of CRC. In combination with this case-control study, our results evidence that $\mathrm{G} \rightarrow \mathrm{A}$ mutation in MTHFR rs1801133 increases the availability of 5, 10-methylenetetrahydrofolate for DNA synthesis; thus, this SNP may be a protective factor of CRC. 
Table 3: The frequencies of $M T H F R$ rs3753584 $\mathrm{T}>\mathrm{C}$, rs9651118 $\mathrm{T}>\mathrm{C}$, rs1801133 $\mathrm{G}>\mathrm{A}$, rs4846048 $\mathrm{A}>\mathrm{G}$ and $\mathrm{rs} 4845882$ G>A polymorphisms in colorectal cancer patients and controls

\begin{tabular}{|c|c|c|c|c|c|c|c|c|}
\hline \multirow[t]{2}{*}{ Genotype } & \multicolumn{2}{|c|}{$\begin{array}{l}\text { Colorectal cancer } \\
\qquad(\mathrm{n}=\mathbf{3 8 7})\end{array}$} & \multirow{2}{*}{$\begin{array}{c}\begin{array}{c}\text { Colon } \\
\text { cancer }\end{array} \\
\mathbf{n}\end{array}$} & \multirow{2}{*}{$\frac{(n=169)}{\%}$} & \multicolumn{2}{|c|}{$\begin{array}{c}\text { Rectum } \\
\text { cancer } \\
(n=218)\end{array}$} & \multirow{2}{*}{$\begin{array}{c}\text { Controls } \\
n\end{array}$} & \multirow{2}{*}{$\begin{array}{c}(\mathrm{n}=1,536) \\
\%\end{array}$} \\
\hline & $\mathbf{n}$ & $\%$ & & & $\mathbf{n}$ & $\%$ & & \\
\hline \multicolumn{9}{|c|}{ MTHFR $\mathrm{rs} 3753584 \mathrm{~T}>\mathrm{C}$} \\
\hline TT & 287 & 74.93 & 131 & 78.44 & 156 & 72.22 & 1211 & 79.00 \\
\hline $\mathrm{TC}$ & 89 & 23.24 & 31 & 18.56 & 58 & 26.85 & 309 & 20.16 \\
\hline $\mathrm{CC}$ & 7 & 1.83 & 5 & 2.99 & 2 & 0.93 & 13 & 0.85 \\
\hline $\mathrm{CT}+\mathrm{CC}$ & 96 & 25.07 & 36 & 21.56 & 60 & 27.78 & 322 & 21.00 \\
\hline $\mathrm{TT}+\mathrm{CT}$ & 376 & 98.17 & 162 & 97.01 & 214 & 99.07 & 1,520 & 99.15 \\
\hline $\mathrm{CC}$ & 7 & 1.83 & 5 & 2.99 & 2 & 0.93 & 13 & 0.85 \\
\hline $\mathrm{C}$ allele & 103 & 13.45 & 41 & 12.28 & 62 & 14.35 & 335 & 10.93 \\
\hline \multicolumn{9}{|c|}{ MTHFR rs9651118 T>C } \\
\hline TT & 131 & 34.20 & 56 & 33.53 & 75 & 34.72 & 578 & 37.70 \\
\hline $\mathrm{TC}$ & 188 & 49.09 & 79 & 47.31 & 109 & 50.46 & 749 & 48.86 \\
\hline $\mathrm{CC}$ & 64 & 16.71 & 32 & 19.16 & 32 & 14.81 & 206 & 13.44 \\
\hline $\mathrm{TC}+\mathrm{CC}$ & 252 & 65.80 & 111 & 66.47 & 141 & 65.28 & 955 & 62.30 \\
\hline $\mathrm{TT}+\mathrm{TC}$ & 319 & 83.29 & 135 & 80.84 & 184 & 85.19 & 1,327 & 86.56 \\
\hline $\mathrm{CC}$ & 64 & 16.71 & 32 & 19.16 & 32 & 14.81 & 206 & 13.44 \\
\hline $\mathrm{C}$ allele & 316 & 41.25 & 143 & 42.81 & 173 & 40.05 & 1,161 & 37.87 \\
\hline \multicolumn{9}{|c|}{ MTHFR $\mathrm{rs} 1801133 \mathrm{G}>\mathrm{A}$} \\
\hline GG & 177 & 46.21 & 82 & 49.10 & 95 & 43.98 & 639 & 41.68 \\
\hline GA & 175 & 45.69 & 73 & 43.71 & 102 & 47.22 & 697 & 45.47 \\
\hline AA & 31 & 8.09 & 12 & 7.19 & 19 & 8.80 & 197 & 12.85 \\
\hline $\mathrm{GA}+\mathrm{AA}$ & 206 & 53.79 & 85 & 50.90 & 121 & 56.02 & 894 & 58.32 \\
\hline $\mathrm{GG}+\mathrm{GA}$ & 352 & 91.91 & 155 & 92.81 & 197 & 91.20 & 1,336 & 87.15 \\
\hline AA & 31 & 8.09 & 12 & 7.19 & 19 & 8.80 & 197 & 12.85 \\
\hline A allele & 237 & 30.94 & 97 & 29.04 & 140 & 32.41 & 1,091 & 35.58 \\
\hline \multicolumn{9}{|c|}{ MTHFR $\mathrm{rs} 4846048 \mathrm{~A}>\mathrm{G}$} \\
\hline AA & 308 & 80.42 & 136 & 81.44 & 172 & 79.63 & 1259 & 82.13 \\
\hline $\mathrm{AG}$ & 70 & 18.28 & 30 & 17.96 & 40 & 18.52 & 254 & 16.57 \\
\hline GG & 5 & 1.31 & 1 & 0.60 & 4 & 1.85 & 20 & 1.30 \\
\hline $\mathrm{AG}+\mathrm{GG}$ & 75 & 19.58 & 31 & 18.56 & 44 & 20.37 & 274 & 17.87 \\
\hline $\mathrm{AA}+\mathrm{AG}$ & 378 & 98.69 & 166 & 99.40 & 212 & 98.15 & 1,513 & 98.70 \\
\hline GG & 5 & 1.31 & 1 & 0.60 & 4 & 1.85 & 20 & 1.30 \\
\hline $\mathrm{G}$ allele & 80 & 10.44 & 32 & 9.58 & 48 & 11.11 & 294 & 9.59 \\
\hline
\end{tabular}




\begin{tabular}{|c|c|c|c|c|c|c|c|c|}
\hline \multirow[t]{2}{*}{ Genotype } & \multicolumn{2}{|c|}{$\begin{array}{l}\text { Colorectal cancer } \\
\qquad(\mathrm{n}=\mathbf{3 8 7})\end{array}$} & \multirow{2}{*}{$\begin{array}{c}\begin{array}{c}\text { Colon } \\
\text { cancer }\end{array} \\
\mathbf{n}\end{array}$} & \multirow{2}{*}{$\begin{array}{c}(\mathrm{n}=169) \\
\% \frac{1}{0}\end{array}$} & \multirow{2}{*}{$\begin{array}{c}\begin{array}{c}\text { Rectum } \\
\text { cancer } \\
(\mathrm{n}=\mathbf{2 1 8})\end{array} \\
\mathrm{n}\end{array}$} & \multirow[b]{2}{*}{$\%$} & \multirow{2}{*}{$\begin{array}{c}\text { Controls } \\
\mathbf{n}\end{array}$} & \multirow{2}{*}{$\frac{(n=1,536}{\%}$} \\
\hline & $\mathbf{n}$ & $\%$ & & & & & & \\
\hline \multicolumn{9}{|c|}{ MTHFR $\mathrm{rs} 4845882 \mathrm{G}>\mathrm{A}$} \\
\hline GG & 228 & 59.53 & 103 & 61.68 & 125 & 57.87 & 956 & 62.36 \\
\hline GA & 129 & 33.68 & 54 & 32.34 & 75 & 34.72 & 512 & 33.40 \\
\hline $\mathrm{AA}$ & 26 & 6.79 & 10 & 5.99 & 16 & 7.41 & 65 & 4.24 \\
\hline $\mathrm{GA}+\mathrm{AA}$ & 155 & 40.47 & 64 & 38.32 & 91 & 42.13 & 577 & 37.64 \\
\hline $\mathrm{GG}+\mathrm{GA}$ & 357 & 93.21 & 157 & 94.01 & 200 & 92.59 & 1,468 & 95.76 \\
\hline $\mathrm{AA}$ & 26 & 6.79 & 10 & 5.99 & 16 & 7.41 & 65 & 4.24 \\
\hline A allele & 181 & 23.63 & 74 & 22.16 & 107 & 24.77 & 642 & 20.94 \\
\hline
\end{tabular}

Table 4: Overall and stratified analyses of MTHFR rs3753584 T>C, rs9651118 T>C, rs1801133 G $>A$, rs4846048 A $>$ G and $\mathbf{r s 4 8 4 5 8 2} \mathrm{G}>\mathrm{A}$ polymorphisms with colorectal cancer

\begin{tabular}{|c|c|c|c|c|c|c|c|c|c|c|c|c|}
\hline \multirow[t]{2}{*}{ Genotype } & \multicolumn{4}{|c|}{$\begin{array}{c}\text { Overall } \\
\text { colorectal } \\
\text { cancer } \\
\text { cases } \\
(\mathbf{n}=387)\end{array}$} & \multicolumn{4}{|c|}{$\begin{array}{l}\text { Colon cancer } \\
\quad(\mathrm{n}=169)\end{array}$} & \multicolumn{4}{|c|}{$\begin{array}{l}\text { Rectum cancer } \\
\quad(\mathrm{n}=218)\end{array}$} \\
\hline & $\begin{array}{c}\text { Crude OR } \\
(95 \% \mathrm{CI})\end{array}$ & $P$ & $\begin{array}{c}\text { Adjusted } \mathrm{OR}^{\mathrm{a}} \\
\quad(\mathbf{9 5} \% \mathrm{CI})\end{array}$ & $P$ & $\begin{array}{c}\text { Crude OR } \\
(95 \% \mathrm{CI})\end{array}$ & $P$ & $\begin{array}{c}\text { Adjusted } \mathrm{OR}^{\mathrm{a}} \\
\quad(\mathbf{9 5} \% \mathrm{CI})\end{array}$ & $P$ & Crude OR $(95 \% \mathrm{Cl})$ & $P$ & $\begin{array}{c}\text { Adjusted } \mathrm{OR}^{\mathrm{a}} \\
\quad(95 \% \mathrm{CI})\end{array}$ & $P$ \\
\hline \multicolumn{13}{|l|}{$\begin{array}{l}\text { MTHFR } \\
\text { rs3753584 } \\
\text { T>C }\end{array}$} \\
\hline $\begin{array}{l}\text { Additive } \\
\text { model }\end{array}$ & $\begin{array}{c}1.20(0.92- \\
1.57)\end{array}$ & 0.180 & $1.20(0.92-1.58)$ & 0.178 & $\begin{array}{c}0.92(0.61- \\
1.38)\end{array}$ & 0.674 & $0.92(0.61-1.38)$ & 0.679 & $1.44(1.04-2.00)$ & 0.028 & $1.44(1.04-2.00)$ & 0.030 \\
\hline $\begin{array}{l}\text { Homozygote } \\
\text { model }\end{array}$ & $\begin{array}{c}2.25(0.89- \\
5.68)\end{array}$ & 0.087 & $2.34(0.92-5.93)$ & 0.074 & $\begin{array}{l}3.51(1.23- \\
10.01)\end{array}$ & 0.019 & $3.63(1.27-10.38)$ & 0.016 & $1.18(0.26-5.29)$ & 0.827 & $1.20(0.27-5.38)$ & 0.815 \\
\hline $\begin{array}{l}\text { Dominant } \\
\text { model }\end{array}$ & $\begin{array}{c}1.26(0.97- \\
1.63)\end{array}$ & 0.086 & $1.26(0.97-1.64)$ & 0.081 & $\begin{array}{c}1.03(0.70- \\
1.53)\end{array}$ & 0.867 & $1.04(0.70-1.53)$ & 0.852 & $1.45(1.05-2.00)$ & 0.025 & $1.44(1.04-2.00)$ & 0.026 \\
\hline $\begin{array}{l}\text { Recessive } \\
\text { model }\end{array}$ & $\begin{array}{c}2.18(0.86- \\
5.49)\end{array}$ & 0.100 & $2.26(0.89-5.73)$ & 0.085 & $\begin{array}{l}3.61(1.27- \\
10.26)\end{array}$ & 0.016 & $3.74(1.31-10.64)$ & 0.014 & $1.09(0.25-4.88)$ & 0.908 & $1.11(0.25-4.97)$ & 0.894 \\
\hline \multicolumn{13}{|l|}{$\begin{array}{l}\text { MTHFR } \\
\text { rs9651118 } \\
\text { T>C }\end{array}$} \\
\hline $\begin{array}{l}\text { Additive } \\
\text { model }\end{array}$ & $\begin{array}{c}1.08(0.84- \\
1.38)\end{array}$ & 0.539 & $1.08(0.85-1.39)$ & 0.528 & $\begin{array}{c}1.06(0.74- \\
1.51)\end{array}$ & 0.762 & $1.06(0.75-1.52)$ & 0.734 & $1.10(0.80-1.50)$ & 0.556 & $1.10(0.80-1.50)$ & 0.552 \\
\hline $\begin{array}{l}\text { Homozygote } \\
\text { model }\end{array}$ & $\begin{array}{c}1.34(0.95- \\
1.87)\end{array}$ & 0.091 & $1.36(0.97-1.90)$ & 0.077 & $\begin{array}{c}1.56(0.98- \\
2.47)\end{array}$ & 0.060 & $1.56(0.99-2.48)$ & 0.057 & $1.17(0.75-1.82)$ & 0.481 & $1.22(0.78-1.90)$ & 0.390 \\
\hline $\begin{array}{l}\text { Dominant } \\
\text { model }\end{array}$ & $\begin{array}{c}1.16(0.92- \\
1.47)\end{array}$ & 0.205 & $1.17(0.93-1.48)$ & 0.190 & $\begin{array}{c}1.20(0.86- \\
1.68)\end{array}$ & 0.290 & $1.21(0.86-1.69)$ & 0.275 & $1.14(0.84-1.53)$ & 0.397 & $1.15(0.85-1.55)$ & 0.368 \\
\hline $\begin{array}{l}\text { Recessive } \\
\text { model }\end{array}$ & $\begin{array}{c}1.29(0.95- \\
1.76)\end{array}$ & 0.100 & $1.31(0.96-1.78)$ & 0.084 & $\begin{array}{c}1.53(1.01- \\
2.31)\end{array}$ & 0.044 & $1.53(1.01-2.31)$ & 0.044 & $1.12(0.75-1.68)$ & 0.581 & $1.16(0.77-1.74)$ & 0.471 \\
\hline \multicolumn{13}{|l|}{$\begin{array}{l}\text { MTHFR } \\
\text { rs1801133 } \\
G>A\end{array}$} \\
\hline $\begin{array}{l}\text { Additive } \\
\text { model }\end{array}$ & $\begin{array}{c}0.89(0.71- \\
1.13)\end{array}$ & 0.331 & $0.89(0.70-1.12)$ & 0.322 & $\begin{array}{c}0.80(0.58- \\
1.12)\end{array}$ & 0.188 & $0.80(0.57-1.11)$ & 0.183 & $0.97(0.72-1.31)$ & 0.834 & $0.97(0.72-1.30)$ & 0.817 \\
\hline $\begin{array}{l}\text { Homozygote } \\
\text { model }\end{array}$ & $\begin{array}{c}0.56(0.37- \\
0.84)\end{array}$ & 0.006 & $0.56(0.37-0.84)$ & 0.005 & $\begin{array}{l}0.47(0.25- \\
0.87)\end{array}$ & 0.017 & $0.46(0.25-0.86)$ & 0.015 & $0.64(0.38-1.07)$ & 0.089 & $0.64(0.38-1.07)$ & 0.090 \\
\hline $\begin{array}{l}\text { Dominant } \\
\text { model }\end{array}$ & $\begin{array}{c}0.83(0.66- \\
1.04)\end{array}$ & 0.109 & $0.83(0.66-1.04)$ & 0.106 & $\begin{array}{c}0.74(0.54- \\
1.02)\end{array}$ & 0.066 & $0.74(0.54-1.02)$ & 0.063 & $0.91(0.68-1.21)$ & 0.522 & $0.91(0.68-1.21)$ & 0.512 \\
\hline $\begin{array}{l}\text { Recessive } \\
\text { model }\end{array}$ & $\begin{array}{c}0.60(0.40- \\
0.89)\end{array}$ & 0.011 & $0.60(0.40-0.89)$ & 0.011 & $\begin{array}{c}0.53(0.29- \\
0.96)\end{array}$ & 0.037 & $0.52(0.28-0.95)$ & 0.035 & $0.65(0.40-1.07)$ & 0.092 & $0.66(0.40-1.08)$ & 0.095 \\
\hline
\end{tabular}

(Continued) 


\begin{tabular}{|c|c|c|c|c|c|c|c|c|c|c|c|c|}
\hline \multirow[t]{2}{*}{ Genotype } & \multicolumn{4}{|c|}{$\begin{array}{c}\text { Overall } \\
\text { colorectal } \\
\text { cancer } \\
\text { cases } \\
(\mathrm{n}=387)\end{array}$} & \multicolumn{4}{|c|}{$\begin{array}{c}\text { Colon cancer } \\
(\mathrm{n}=169)\end{array}$} & \multicolumn{4}{|c|}{$\begin{array}{l}\text { Rectum cancer } \\
\quad(\mathrm{n}=218)\end{array}$} \\
\hline & $\begin{array}{c}\text { Crude OR } \\
\text { (95\%CI) }\end{array}$ & $P$ & $\begin{array}{l}\text { Adjusted } \mathrm{OR}^{\mathrm{a}} \\
(95 \% \mathrm{CI})\end{array}$ & $P$ & $\begin{array}{c}\text { Crude OR } \\
(95 \% \mathrm{CI})\end{array}$ & $P$ & $\begin{array}{c}\text { Adjusted } \mathrm{OR}^{\mathrm{a}} \\
\quad(\mathbf{9 5} \% \mathrm{CI})\end{array}$ & $P$ & Crude OR $(95 \% \mathrm{CI})$ & $P$ & $\begin{array}{c}\text { Adjusted } \mathrm{OR}^{\mathrm{a}} \\
\quad(95 \% \mathrm{CI})\end{array}$ & $P$ \\
\hline \multicolumn{13}{|l|}{$\begin{array}{l}M T H F R \\
\begin{array}{l}\text { rs } 4846048 \\
\mathrm{~A}>\mathrm{G}\end{array}\end{array}$} \\
\hline $\begin{array}{l}\text { Additive } \\
\text { model }\end{array}$ & $\begin{array}{c}1.12(0.83- \\
1.49)\end{array}$ & 0.466 & $1.11(0.83-1.49)$ & 0.474 & $\begin{array}{c}1.08(0.71- \\
1.64)\end{array}$ & 0.717 & $1.08(0.71-1.63)$ & 0.735 & $1.14(0.79-1.65)$ & 0.480 & $1.14(0.79-1.66)$ & 0.482 \\
\hline $\begin{array}{l}\text { Homozygote } \\
\text { model }\end{array}$ & $\begin{array}{l}1.01(0.38- \\
2.72)\end{array}$ & 0.982 & $0.98(0.36-2.63)$ & 0.961 & $\begin{array}{c}0.46(0.06- \\
3.43)\end{array}$ & 0.447 & $0.46(0.06-3.48)$ & 0.454 & $1.45(0.49-4.30)$ & 0.501 & $1.36(0.45-4.07)$ & 0.583 \\
\hline $\begin{array}{l}\text { Dominant } \\
\text { model }\end{array}$ & $\begin{array}{c}1.12(0.84- \\
1.49)\end{array}$ & 0.439 & 1.11(0.84-1.48) & 0.457 & $\begin{array}{c}1.05(0.69- \\
1.58)\end{array}$ & 0.826 & $1.04(0.69-1.58)$ & 0.839 & $1.18(0.82-1.68)$ & 0.373 & $1.17(0.82-1.67)$ & 0.393 \\
\hline $\begin{array}{l}\text { Recessive } \\
\text { model }\end{array}$ & $\begin{array}{c}1.00(0.37- \\
2.68)\end{array}$ & 1.000 & $0.96(0.36-2.59)$ & 0.938 & $\begin{array}{c}0.46(0.06- \\
3.42)\end{array}$ & 0.445 & $0.46(0.06-3.45)$ & 0.450 & $1.43(0.48-4.22)$ & 0.519 & $1.33(0.45-3.98)$ & 0.609 \\
\hline \multicolumn{13}{|l|}{$\begin{array}{l}\text { MTHFR } \\
\text { rs4845882 } \\
\mathrm{G}>\mathrm{A}\end{array}$} \\
\hline $\begin{array}{l}\text { Additive } \\
\text { model }\end{array}$ & $\begin{array}{c}1.04(0.82- \\
1.33)\end{array}$ & 0.741 & $1.05(0.82-1.33)$ & 0.720 & $\begin{array}{c}0.96(0.68- \\
1.36)\end{array}$ & 0.832 & $0.97(0.68-1.36)$ & 0.843 & $1.11(0.82-1.50)$ & 0.517 & $1.11(0.82-1.51)$ & 0.508 \\
\hline $\begin{array}{l}\text { Homozygote } \\
\text { model }\end{array}$ & $\begin{array}{l}1.65(1.03- \\
2.66)\end{array}$ & 0.039 & $1.64(1.02-2.65)$ & 0.043 & $\begin{array}{c}1.41(0.70- \\
2.82)\end{array}$ & 0.338 & $1.42(0.71-2.85)$ & 0.326 & $1.86(1.04-3.31)$ & 0.035 & $1.79(1.00-3.20)$ & 0.050 \\
\hline $\begin{array}{l}\text { Dominant } \\
\text { model }\end{array}$ & $\begin{array}{c}1.13(0.90- \\
1.42)\end{array}$ & 0.308 & $1.13(0.90-1.42)$ & 0.300 & $\begin{array}{c}1.03(0.74- \\
1.43)\end{array}$ & 0.862 & $1.03(0.74-1.44)$ & 0.845 & $1.21(0.90-1.61)$ & 0.204 & $1.20(0.90-1.61)$ & 0.211 \\
\hline $\begin{array}{l}\text { Recessive } \\
\text { model }\end{array}$ & $\begin{array}{c}1.65(1.03- \\
2.63)\end{array}$ & 0.038 & $1.63(1.01-2.60)$ & 0.044 & $\begin{array}{c}1.44(0.73- \\
2.86)\end{array}$ & 0.299 & $1.45(0.73-2.88)$ & 0.290 & $1.81(1.03-3.18)$ & 0.041 & $1.73(0.98-3.07)$ & 0.059 \\
\hline
\end{tabular}

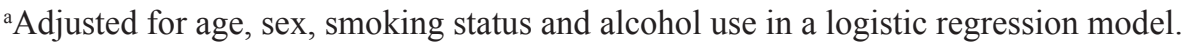

To the best of our knowledge, it was the first epidemiological study to explore the relationship of MTHFR rs4845882 G>A polymorphism with $\mathrm{CRC}$ risk. MTHFR rs4845882 $\mathrm{G}>\mathrm{A}$ is linkage disequilibrium (LD) with rs1801131 (1298 A>C). Several metaanalyses suggested that MTHFR $\operatorname{rs} 1801131 \mathrm{~A}>\mathrm{C}$ polymorphism affected risk of CRC in Asians [31, 32]. In the present study, we found MTHFR rs 4845882 $\mathrm{G}>\mathrm{A}$ polymorphism may be associated with the development of CRC. Since MTHFR rs $4845882 \mathrm{G}>\mathrm{A}$ and rs1801131 A $>$ C are in strong LD $\left(r^{2}=0.935\right.$, http://gvs. gs.washington.edu/GVS147/LDpairwiseR2.jsp?GET TAYLORGRAM=1491876387794), the function of rs4845882 G $>$ A may be affected by rs $1801131 \mathrm{~A}>\mathrm{C}$.

A study reported that CC genotype of MTHFR rs 9651118 conferred a reduced risk of breast cancer compared to TT genotype in a Chinese population [33]. Swartz et al. found MTHFR rs9651118 T $>\mathrm{C}$ polymorphism may be correlated with the decreased risk of lung cancer in Caucasians [34]. In our previous study, we found there was null association between MTHFR rs9651118 $\mathrm{T}>\mathrm{C}$ polymorphism and esophageal squamous cell carcinoma in a Chinese Han population [21]. However, in this study, we found that rs 9651118 CC genotype was relevant to increased risk of colon cancer. Rs9651118 T>C polymorphism is located on the intron of MTHFR gene. The function of this polymorphism is not well known. It was reported that MTHFR rs9651118 TT genotype elevated the level of Hcy compared with CC genotype [35]. In the future, more functional studies are required to identify the real biological effect of MTHFR rs9651118 T $>\mathrm{C}$ polymorphism on the etiology of CRC.

In a subgroup analysis by the region of CRC, MTHFR rs3753584 $\mathrm{T}>\mathrm{C}$ polymorphism was associated with the risk of colon and rectum cancer. Although the function of MTHFR rs3753584 $\mathrm{T}>\mathrm{C}$ polymorphism was not identified, a significantly increased risk of lung cancer was found for the variant allele carriers of this polymorphism, compared with individuals with wild homozygote [36]. In this study, we also found that C allele of MTHFR rs3753584 was probably correlated with an increased risk of colon and rectum cancer, which was consistent with the findings of those previous study.

Like all case-control studies, this study has some limitations. First, demographic variables and risk factor information only focused on age, sex, smoking and alcohol consumption. And other lifestyles were not collected, which might increase the possibility of confounding from environmental risk factors. Second, the source of non-cancer controls was hospital-based; which might not well represent the whole Chinese population. Third, in a stratified analysis by the region of $\mathrm{CRC}$, the relatively small sample size may decrease the power of the results. Finally, these findings should be interpreted with very caution because the participants were only enrolled from Chinese Han population. Thus, the results may not permit extrapolation to other ethnicities. 
In summary, the tagging polymorphisms in MTHFR gene (rs3753584 $\mathrm{T}>\mathrm{C}$, rs9651118 $\mathrm{T}>\mathrm{C}$ and $\mathrm{rs} 4845882$ $\mathrm{G}>\mathrm{A}$ ) are associated with an increased risk of $\mathrm{CRC}$. However, MTHFR rs1801133 G>A polymorphism confers a decreased risk to CRC. Our findings suggest that further validation studies are needed.

\section{MATERIALS AND METHODS}

\section{Study population and patient selection}

The study population consisted of 387 sporadic, consecutive CRC patients from the Department of General Surgery, Fujian Medical University Union Hospital (Fuzhou, China) between October 2014 and May 2016. Histological report confirmed the diagnosis of CRC. Noncancer controls $(n=1,536)$ were randomly recruited from the Fujian Medical University Union Hospital (Fuzhou, China) and the Affiliated People's Hospital of Jiangsu University (Zhenjiang, China) between October 2014 and November 2016. The CRC patients and controls were matched for age, sex and residential area (Eastern China). Every participant was informed the aim of the present study and signed an informed consent. The protocol of the study was obtained by the institutional ethics committee of Fujian Medical University and Jiangsu University. Additionally, in this case-control study, we conformed to the principles of Declaration of Helsinki.

\section{Data collection}

Every participant was personally questioned and answered a questionnaire regarding age, sex, the status of cigarette smoking, and alcohol consumption. Participants who drink more than thrice/week for $>6$ months and smoke at least one cigarette/day over 1 year were considered positive. Clinical characteristics, such as pathological stage and tumor site, were obtained from the medical records (Table 1).

\section{Selection of tagging SNPs}

The tagging SNPs of MTHFR gene [30.4 Kbp spanning from 11780730 to 11811103 in chromosome 1 (upstream and downstream of the gene extending 5000 bases, respectively)] were analyzed and selected from the data of Chinese Han individuals in Beijing (CHB) via the HapMap Project (http://hapmap.ncbi.nlm.nih.gov/index. html.en) [20]. The detailed process and criterion were described previously [21]. The information of selected MTHFR tagging SNPs is presented in Table 2.

\section{DNA extraction and genotyping}

Ethylenediamine tetraacetic acid-anticoagulated intravenous blood was donated by every participant. Using the Promega DNA Blood Mini Kit (Promega, Madison,
USA), genomic DNA was extracted from peripheral lymphocytes by the standard experimental protocol.

A custom-by-design 48-Plex SNPscan Kit (Genesky Biotechnologies Inc., Shanghai, China), double ligation and multiplex fluorescence PCR [22], was performed to identify the genotypes of MTHFR $\operatorname{rs} 3753584 \mathrm{~T}>\mathrm{C}$, rs9651118 T $>$ C, rs1801133 G $>A$, rs4846048 $\mathrm{A}>\mathrm{G}$ and rs4845882 $\mathrm{G}>\mathrm{A}$ polymorphisms as described in previous studies [23, 24]. A total of 77 samples were randomly selected and were tested again for quality control. Based on $4 \%$ of duplicated samples in this study, the accordance rates were $100 \%$.

\section{Statistical analysis}

We used SAS statistical software, version 9.4 (SAS Institute, Cary, NC) for data analysis and a $P$ $<0.05$ (two-tailed) was considered to be a statistical significance. The quantitative variables were expressed as means \pm standard deviation (SD). Student's t-test was harnessed to evaluate the difference of age between CRC patients and controls. Additionally, we used $\chi^{2}$ test to examine the differences in age, sex, smoking status, alcohol consumption and the frequencies of genotype between patients with $\mathrm{CRC}$ and controls. The HardyWeinberg equilibrium (HWE) equation was used to assess whether the proportion of MTHFR tagging SNPs genotypes obtained was in accordance with the expected value. An online calculator (http://ihg.gsf.de/cgi-bin/ hw/hwa1.pl) was harnessed to calculate the $P$ value of HWE [25]. The relationship of MTHFR $\operatorname{rs} 3753584 \mathrm{~T}>\mathrm{C}$, rs9651118 T $>$ C, rs1801133 G>A, rs4846048 A $>$ G and rs4845882 $\mathrm{G}>\mathrm{A}$ genotypes with $\mathrm{CRC}$ risk was estimated by crude/adjusted odds ratios (ORs) and $95 \%$ confidence intervals (CIs). The power of the present study $(\alpha=0.05)$ was evaluated by the Power and Sample Size Calculator (http://biostat.mc.vanderbilt.edu/twiki/bin/view/Main/ PowerSampleSize).

\section{ACKNOWLEDGMENTS}

We appreciate all subjects who participated in this study. We wish to thank Dr. Yan Liu (Genesky Biotechnologies Inc., Shanghai, China) for technical support.

\section{CONFLICTS OF INTEREST}

The authors have no potential financial conflicts of interest.

\section{GRANT SUPPORT}

This study was supported by Natural Science Foundation of Universities and colleges of Jiangsu Province (Grant No. 16KJB310002), Senior Talents 
Scientific Research Foundation of Jiangsu University (Grant No. 16JDG066), Critical Patented Project of the Science and Technology Bureau of Fujian Province (Grant No. 2013YZ0002-2), the Special Program for the Development of Strategic Emerging Industries of Fujian Province (Grant No. 13YZ0201) and the Natural Science Foundation of Fujian Province (Grant No. 2015J01435).

\section{REFERENCES}

1. Chen W, Zheng R, Baade PD, Zhang S, Zeng H, Bray F, Jemal A, Yu XQ, He J. Cancer statistics in China, 2015. CA Cancer J Clin. 2016; 66:115-132.

2. Chen W, Zheng R, Zeng H, Zhang S, He J. Annual report on status of cancer in China, 2011. Chin J Cancer Res. 2015; 27:2-12.

3. Weitz J, Koch M, Debus J, Hohler T, Galle PR, Buchler MW. Colorectal cancer. Lancet. 2005; 365:153-165.

4. van Duijnhoven FJ, Bueno-De-Mesquita HB, Ferrari P, Jenab M, Boshuizen HC, Ros MM, Casagrande C, Tjonneland A, Olsen A, Overvad K, Thorlacius-Ussing O, Clavel-Chapelon F, Boutron-Ruault MC, et al. Fruit, vegetables, and colorectal cancer risk: the European Prospective Investigation into Cancer and Nutrition. Am J Clin Nutr. 2009; 89:1441-1452.

5. Gerber M. Background review paper on total fat, fatty acid intake and cancers. Ann Nutr Metab. 2009; 55:140-161.

6. Xu M, Chen YM, Huang J, Fang YJ, Huang WQ, Yan B, Lu MS, Pan ZZ, Zhang CX. Flavonoid intake from vegetables and fruits is inversely associated with colorectal cancer risk: a case-control study in China. Br J Nutr. 2016; 116:1275-1287.

7. Nagle CM, Wilson LF, Hughes MC, Ibiebele TI, Miura K, Bain CJ, Whiteman DC, Webb PM. Cancers in Australia in 2010 attributable to inadequate consumption of fruit, non-starchy vegetables and dietary fibre. Aust N Z J Public Health. 2015; 39:422-428.

8. Weigl K, Jansen L, Chang-Claude J, Knebel P, Hoffmeister M, Brenner H. Family history and the risk of colorectal cancer: the importance of patients' history of colonoscopy. Int J Cancer. 2016; 139:2213-2220.

9. Lowery JT, Ahnen DJ, Schroy PC 3rd, Hampel H, Baxter N, Boland CR, Burt RW, Butterly L, Doerr M, Doroshenk M, Feero WG, Henrikson N, Ladabaum U, et al. Understanding the contribution of family history to colorectal cancer risk and its clinical implications: a state-of-the-science review. Cancer. 2016; 122:2633-2645.

10. Kennedy DA, Stern SJ, Matok I, Moretti ME, Sarkar M, Adams-Webber T, Koren G. Folate intake, MTHFR polymorphisms, and the risk of colorectal cancer: a systematic review and meta-analysis. J Cancer Epidemiol. 2012; 2012:952508.

11. Kato I, Dnistrian AM, Schwartz M, Toniolo P, Koenig K, Shore RE, Akhmedkhanov A, Zeleniuch-Jacquotte A, Riboli
E. Serum folate, homocysteine and colorectal cancer risk in women: a nested case-control study. Br J Cancer. 1999; 79:1917-1922.

12. Martinez ME, Giovannucci E, Jiang R, Henning SM, Jacobs ET, Thompson P, Smith-Warner SA, Alberts DS. Folate fortification, plasma folate, homocysteine and colorectal adenoma recurrence. Int J Cancer. 2006; 119:1440-1446.

13. Giovannucci E, Wu K. Cancers of the colon and rectum. In: Cancer epidemiology and prevention, 3rd ed. New York: Oxford University Press. 2006:809-829.

14. McGlynn AP, Wasson GR, O'Reilly SL, McNulty H, Downes CS, Chang CK, Hoey L, Molloy AM, Ward M, Strain JJ, McKerr G, Weir DG, Scott JM. Low colonocyte folate is associated with uracil misincorporation and global DNA hypomethylation in human colorectum. J Nutr. 2013; 143:27-33.

15. Wright AJ, Dainty JR, Finglas PM. Folic acid metabolism in human subjects revisited: potential implications for proposed mandatory folic acid fortification in the UK. Br J Nutr. 2007; 98:667-675.

16. Lurje G, Zhang W, Yang D, Groshen S, Hendifar AE, Husain H, Nagashima F, Chang HM, Fazzone W, Ladner RD, Pohl A, Ning Y, Iqbal S, et al. Thymidylate synthase haplotype is associated with tumor recurrence in stage II and stage III colon cancer. Pharmacogenet Genomics. 2008; 18:161-168.

17. Scott J, Weir D. Folate/vitamin B12 inter-relationships. Essays Biochem. 1994; 28:63-72.

18. Rosenblatt DS. Methylenetetrahydrofolate reductase. Clin Invest Med. 2001; 24:56-59.

19. Sameer AS, Shah ZA, Nissar S, Mudassar S, Siddiqi MA. Risk of colorectal cancer associated with the methylenetetrahydrofolate reductase (MTHFR) C677T polymorphism in the Kashmiri population. Genet Mol Res. 2011; 10:1200-1210.

20. Carlson CS, Eberle MA, Kruglyak L, Nickerson DA. Mapping complex disease loci in whole-genome association studies. Nature. 2004; 429:446-452.

21. Tang W, Zhang S, Qiu H, Wang L, Sun B, Yin J, Gu H. Genetic variations in MTHFR and esophageal squamous cell carcinoma susceptibility in Chinese Han population. Med Oncol. 2014; 31:915.

22. Yin J, Wang X, Wei J, Wang L, Shi Y, Zheng L, Tang W, Ding G, Liu C, Liu R, Chen S, Xu Z, Gu H. Interleukin 12B rs3212227 $\mathrm{T}>\mathrm{G}$ polymorphism was associated with an increased risk of gastric cardiac adenocarcinoma in a Chinese population. Dis Esophagus. 2015; 28:291-298.

23. Zheng L, Yin J, Wang L, Wang X, Shi Y, Shao A, Tang W, Ding G, Liu C, Chen S, Gu H. Interleukin 1B rs16944 $\mathrm{G}>\mathrm{A}$ polymorphism was associated with a decreased risk of esophageal cancer in a Chinese population. Clin Biochem. 2013; 46:1469-1473.

24. Yin J, Wang L, Shi Y, Shao A, Tang W, Wang X, Ding G, Liu C, Chen S, Gu H. Interleukin 17A rs4711998 A>G 
polymorphism was associated with a decreased risk of esophageal cancer in a Chinese population. Dis Esophagus. 2014; 27:87-92.

25. Tang W, Wang Y, Chen S, Lin J, Chen B, Yu S, Chen Y, $\mathrm{Gu}$ H, Kang M. Investigation of cytotoxic T-lymphocyte antigen 4 polymorphisms in gastric cardia adenocarcinoma. Scand J Immunol. 2016; 83:212-218.

26. Langevin SM, Lin D, Matsuo K, Gao CM, Takezaki T, Stolzenberg-Solomon RZ, Vasavi M, Hasan Q, Taioli E. Review and pooled analysis of studies on MTHFR C677T polymorphism and esophageal cancer. Toxicol Lett. 2009; 184:73-80.

27. Teng Z, Wang L, Cai S, Yu P, Wang J, Gong J, Liu Y. The $677 \mathrm{C}>\mathrm{T}$ (rs1801133) polymorphism in the MTHFR gene contributes to colorectal cancer risk: a meta-analysis based on 71 research studies. PLoS One. 2013; 8:e55332.

28. Guo XP, Wang Y, Zhao H, Song SD, Zhou J, Han Y. Association of MTHFR C677T polymorphisms and colorectal cancer risk in Asians: evidence of 12,255 subjects. Clin Transl Oncol. 2014; 16:623-629.

29. Taioli E, Garza MA, Ahn YO, Bishop DT, Bost J, Budai B, Chen K, Gemignani F, Keku T, Lima CS, Le Marchand L, Matsuo K, Moreno V, et al. Meta- and pooled analyses of the methylenetetrahydrofolate reductase (MTHFR) C677T polymorphism and colorectal cancer: a HuGE-GSEC review. Am J Epidemiol. 2009; 170:1207-1221.

30. Frosst P, Blom HJ, Milos R, Goyette P, Sheppard CA, Matthews RG, Boers GJ, den Heijer M, Kluijtmans LA, van den Heuvel LP, Rozen R. A candidate genetic risk factor for vascular disease: a common mutation in methylenetetrahydrofolate reductase. Nat Genet. 1995; 10:111-113.

31. Zhao M, Li X, Xing C, Zhou B. Association of methylenetetrahydrofolate reductase C677T and A1298C polymorphisms with colorectal cancer risk: a meta-analysis. Biomed Rep. 2013; 1:781-791.

32. Zhu XL, Liu ZZ, Yan SX, Wang W, Chang RX, Zhang CY, Guo Y. Association between the MTHFR A1298C polymorphism and risk of cancer: evidence from 265 casecontrol studies. Mol Genet Genomics. 2016; 291:51-63.

33. Lu Q, Jiang K, Li Q, Ji YJ, Chen WL, Xue XH. Polymorphisms in the MTHFR gene are associated with breast cancer risk and prognosis in a Chinese population. Tumour Biol. 2015; 36:3757-3762.

34. Swartz MD, Peterson CB, Lupo PJ, Wu X, Forman MR, Spitz MR, Hernandez LM, Vannucci M, Shete S. Investigating multiple candidate genes and nutrients in the folate metabolism pathway to detect genetic and nutritional risk factors for lung cancer. PLoS One. 2013; 8:e53475.

35. Zhou BS, Bu GY, Li M, Chang BG, Zhou YP. Tagging SNPs in the MTHFR gene and risk of ischemic stroke in a Chinese population. Int J Mol Sci. 2014; 15:8931-8940.

36. Liu H, Jin G, Wang H, Wu W, Liu Y, Qian J, Fan W, Ma H, Miao R, Hu Z, Sun W, Wang Y, Jin L, et al. Association of polymorphisms in one-carbon metabolizing genes and lung cancer risk: a case-control study in Chinese population. Lung Cancer. 2008; 61:21-29. 